\title{
Methodological considerations for the design and implementation of reliable and valid web surveys
}

\author{
Israel Agaku ${ }^{1}$
}

\begin{abstract}
AFFILIATION
1 Department of Oral Health Policy and Epidemiology, Harvard School of Dental Medicine, Harvard University, Boston, United States
\end{abstract}

CORRESPONDENCE TO

Israel Agaku. Department of Oral Health Policy and Epidemiology, Harvard School of Dental Medicine, Harvard University, Boston, MA, United States.

E-mail: iagaku@post.harvard.edu

\section{KEYWORDS}

survey methods, sample size, study design, sample, statistics

Received: 1 September 2021, Accepted: 6 September 2021

Public Health Toxicol 2021;1(1):4

https://doi.org/10.18332/pht/141977

\section{ABSTRACT}

This article examines practical epidemiology principles related to the design and implementation of web surveys. Six practice-oriented items are critically examined: 1) The study question; 2) The target population; 3) Study population needed; 4) Sampling or selecting the participants in a representative manner; 5) Sending the survey invitations in a manner that is efficient, safe, and mitigates bias; and 6) Assessing and enhancing the external validity of collected data. Clearly articulating the study's purpose (including whether there is an intent to create generalizable knowledge) influences the sampling approach: probabilistic or non-probabilistic. Similarly, properly defining the study population (people, place and time) prevents overgeneralization of study findings. Adjustments to sample size may be needed to address different real-world complexities, including multi-purpose surveys with different (possibly un-related outcomes), multiple target populations, subgroup analyses, and cluster sampling. When the sample is being drawn from a sampling frame, efforts must be made to ensure that the frame is complete, current, and correct to reduce under-sampling. The choice of environment in which data collection is hosted is critical; practical considerations include data volume, variety, vulnerability, and the software's capabilities and cost. Although web surveys, in general, are becoming increasingly easier to conduct, good web surveys in contrast are becoming increasingly harder to undertake. Careful consideration should be given to sampling and nonsampling sources of error when designing web surveys to ensure validity and reliability.

\section{INTRODUCTION}

Web-based surveys have gained increased popularity in recent years because of their efficiency in time and $\cos ^{1-}$

${ }^{6}$. This increased resource efficiency, however, potentially comes at the price of decreased precision and validity because of inadequate sampling and low response rates ${ }^{7}$. People are increasingly bombarded with emails; the average American office worker in 2017 received about 120 emails per day, of which only $34 \%$ were opened ${ }^{8}$. A low response rate (or small sample size) does not automatically signal a selection bias problem just as a high response rate (or large sample size) does not automatically guarantee validity (e.g. a large volunteer sample). Yet, the strong potential for bias with web surveys requires careful attention to sampling and non-sampling errors that could threaten their validity?
Despite their increasing adoption and use, there are relatively few resources for public health practitioners on applied epidemiological considerations when conducting web surveys; much of the existing body of knowledge has been limited to comparisons of indexes of performance (e.g. response rates) across survey modes ${ }^{9-13}$. This article presents an overview of web survey design and implementation. Six practice-oriented items are critically examined: 1 ) The study question; 2) The target population; 3) Study population needed; 4) Sampling or selecting the participants in a representative manner; 5) Sending the survey invitations in a manner that is efficient, safe, and mitigates bias; and 6) Assessing and enhancing the external validity of collected data. For each of these items, a broad overview of principles, rather than specifics, is provided to prepare public health 
practitioners to become better acquainted with the design and implementation of web surveys.

This study assumes a list-based, probability web survey where a sampling frame is constructed from an existing directory/register, or other source, with contact information abstracted and used to contact participants. However, several of these principles may also apply to other web survey modes, e.g. pre-recruited panels of internet users. Sample size calculations are assumed to be based on statistical precision of estimates, rather than on the basis of cost.

\section{METHODOLOGICAL APPROACH}

\section{The study question}

Properly articulating the research question can help with critical decision making, including determining whether the question posed can be answered with a cross-sectional design, or whether there is even need to collect primary data (e.g. comparable secondary data sources already exist). Furthermore, it can help with developing appropriate survey instruments that have relevant content and coherent flow. To reduce measurement bias, new survey questions should undergo cognitive testing. Previously validated survey instruments could also be adapted for new information collection activities.

Sometimes, a survey is multi-purpose, with several primary outcomes of interest (and possibly different target populations $)^{14}$. As a general principle, only the minimum amount of data needed should be collected, and no identifying/potentially identifying information should be assessed, except unless it is absolutely critical to the research question. Direct identifiers include any information collected that provides a reasonable basis to identify an individual; examples of such direct identifies include the following 18 categories: name; all geographical subdivisions smaller than state (street, city, county, zip codes or equivalent geocodes, except for initial 3 digits of a zip code); all elements of dates, except year; all ages $>89$ years (unless aggregated into single category of age $>90$ years); telephone and fax number; email, IP address, URL; social security number/passport number; medical record number; health plan beneficiary number; account number; certificate license number; vehicle identification number, device identifiers, and serial number; full face photographs, biometric identifiers; and any other unique identifying number, characteristic or code $\mathrm{e}^{15,16}$. Institute Review Board approval should be sought when direct identifiers or potentially identifying information might be needed or possibly collected; at all times, adequate access controls should be implemented to safeguard protected records (Table 1).

\section{The target population of the survey}

Failure to properly define the target population from which the sample is being drawn could create challenges with making inferences from the sample back to the target population (Figure 1). The target population is determined by the purpose for which data are being collected. In public health, data collection activities are generally classified as either research or public health practice, based on intent to create generalizable knowledge ${ }^{17,18}$. Research activities (e.g. population surveys) have an intent to generalize findings from the studied population to the larger target population (Figure 2). In contrast, activities deemed as public health practice (e.g. focus groups) have no intent to generalize findings beyond the immediate participants studied; the studied population therefore is the same as the target population.

In research activities, the target population could be defined on the basis of different factors, including geographical (e.g. a county), population (e.g. children), administrative (e.g. program directors), occupational/ vocational (e.g. employees or students), or facilities (e.g. clinics). It could also be defined by an event (e.g. birth, death, diagnoses, survivorship, immigration, or emigration), or a behavior (e.g. current tobacco use). Regardless of the criterion used, the epidemiological concepts of person, place, and time should be used to frame the target population. The presence of several target populations within the same survey might have implications for questionnaire design (skip patterns) and sample size calculations (minimum sample size needed).

\section{CASE STUDIES OR PRACTICAL EXAMPLES}

\section{Survey population}

This item touches on issues of precision; a large sample is more precise than a smaller sample (Figure 3). The fundamental approach to sample size calculation does

\section{Table 1. Access controls necessary to ensure protection of participant privacy}

\begin{tabular}{|c|c|c|}
\hline Technical Controls & Physical controls & Administrative controls \\
\hline $\begin{array}{l}\text { - User identification } \\
\text { - Passwords } \\
\text { - Firewall } \\
\text { - Virtual Private Network } \\
\text { (VPN) }\end{array}$ & $\begin{array}{l}\text { - Guards/security officers } \\
\text { - 24-hour maintenance } \\
\text { of video/audio of all data } \\
\text { centers and all offices } \\
\text { - Identification badges } \\
\text { - Key card }\end{array}$ & $\begin{array}{l}\text { - No directly identifying information will be collected (thus, the } \\
\text { Privacy Act does not apply). } \\
\text { - All study personnel have completed training on ethical conduct of } \\
\text { Human Subject Research. } \\
\text { - Methods will be in place to ensure limited access. Data and all } \\
\text { identifying information about respondents will be handled in ways } \\
\text { that prevent unauthorized access at any point during the study. }\end{array}$ \\
\hline
\end{tabular}


Figure 1. The cycle of inference for non-probability versus probability based samples
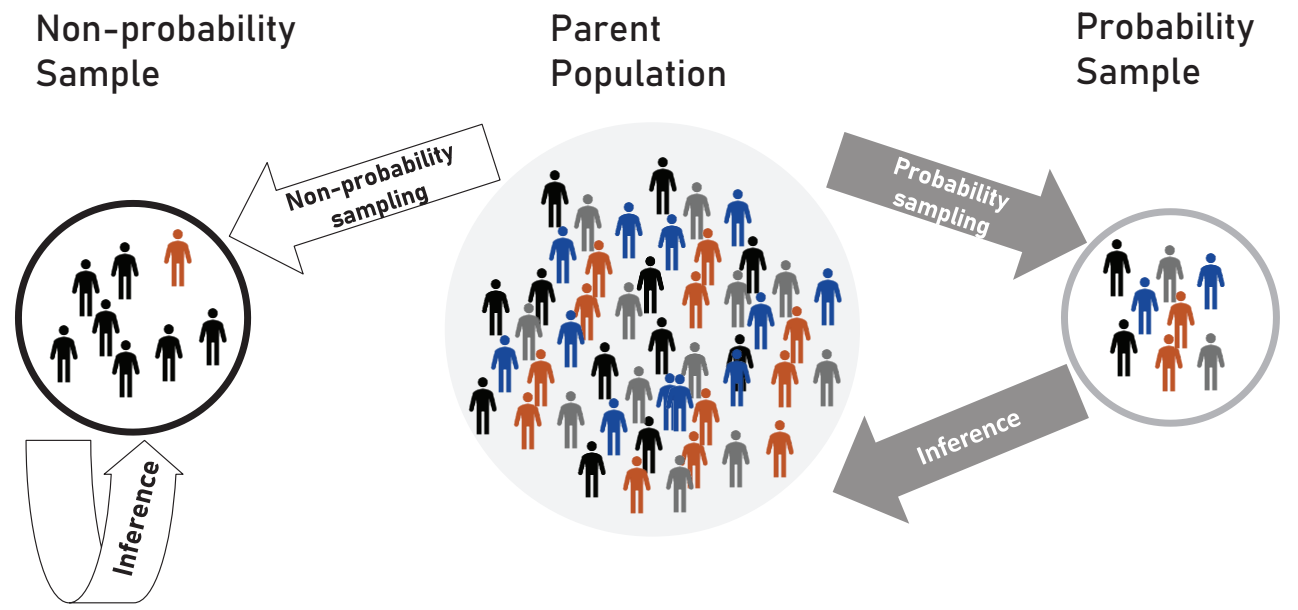

Figure 2. Algorithm for decision making in relation to sampling approach in survey design

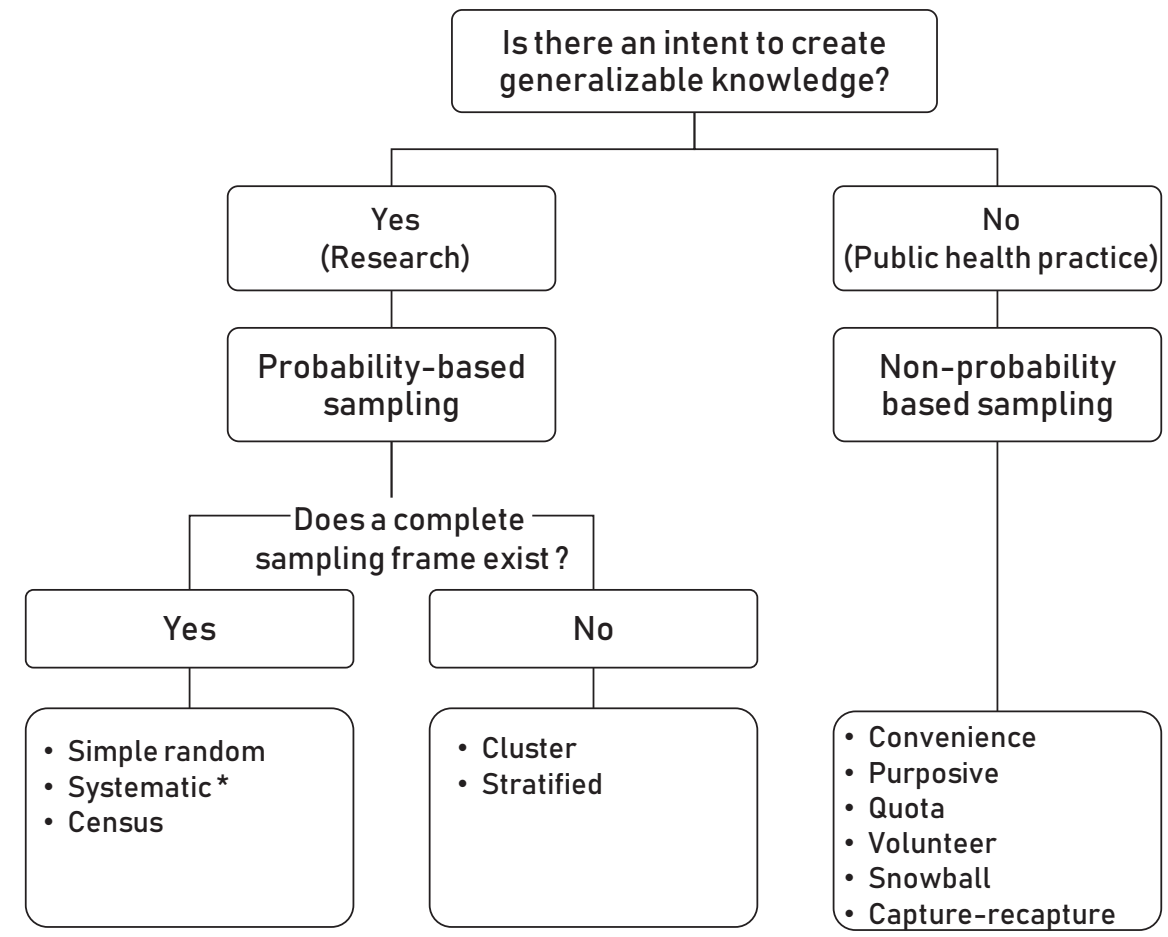

* Systematic sampling is only probability-based when the first individual in a sorted sampling frame is selected at random. In traditional survey modes (e.g. in-person household surveys), a sampling frame may not be needed for systematic sampling (e.g. the interviewer starts from a random house on the street and selects every $n$-th house afterwards). With web-based surveys, however, this cannot be executed without a sampling frame constructed.

not differ between web versus traditional survey modes; however, more stringent assumptions may be needed for web surveys (e.g. smaller anticipated response rate). Questions which investigators might grapple with during sample size calculations include: 'How do I ensure that I can generate reliable estimates for the smallest-sized subgroups of interest in my study?'; 'What if I have several outcomes or target populations within the same survey?'; 'How do I determine reasonable estimates of the different parameters needed for sample size calculation?'.

The following principles are applied when performing sample size calculations:

1. When several key outcomes are being assessed (e.g. a multi-purpose survey), sample size could be computed for each key outcome, and the largest sample size used. Alternatively, a prevalence of $50 \%$ could be used to 
Figure 3. Four hypothetical scenarios demonstrating differences in validity* and precision** using an arbitrarily chosen population parameter (i.e. 'gold standard') of $20 \%$ for prevalence of an unspecified outcome $^{* * *}$

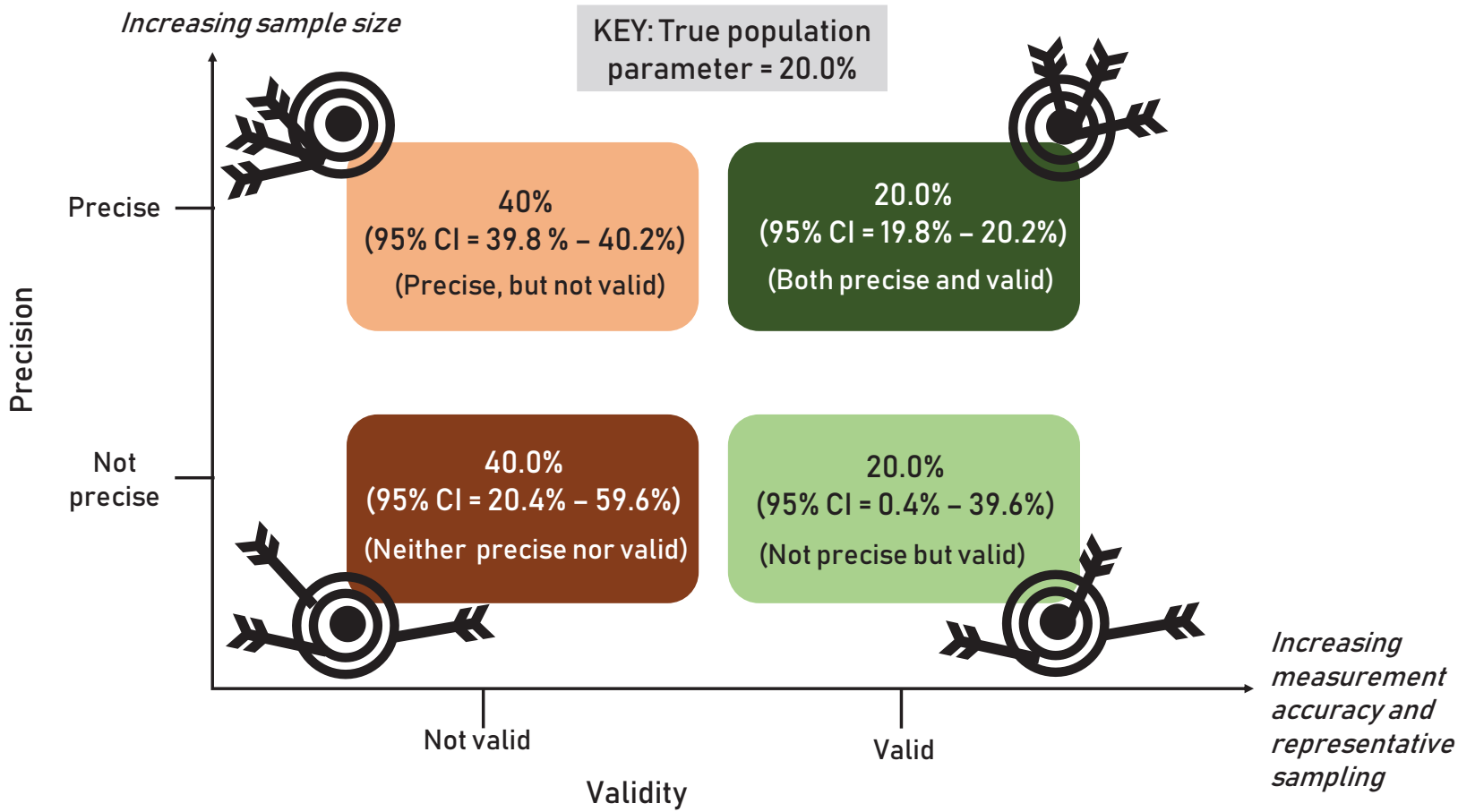

* Validity measures how close an estimate is to the true population parameter (illustrated when an arrow hits the bullseye). ** Precision or reliability measures how close measurements taken are to each other (i.e. reproducibility). An imprecise estimate will have $95 \%$ confidence intervals that are very wide or standard errors (SE) that are very large. A commonly used precision rule to ensure that only reliable estimates are reported is suppressing estimates with relative standard errors greater than $30 \%$ (relative standard error $=\mathrm{SE} / \mathrm{mean}$ ). ${ }^{* * *}$ In the hypothetical scenarios presented in the figure above, SEs of 0.1 and 10 were arbitrarily assumed for precise and imprecise estimates respectively; $95 \%$ confidence intervals were calculated as percentage $\pm 1.96 \times$ SE.

maximize yield.

2. When there are multiple target populations being assessed within the same survey, sample size calculations should be based on the smallest one to ensure that there is adequate precision for the other target population(s).

3. When there are multiple tabulation variables (e.g. sex, race/ethnicity, and census region) and there is a need to ensure precise estimates for all cells of all variables, sample size should be based on the smallest base population (the smallest category of the tabulation variable with the most levels). A cautionary note, the tinier the smallest base population for which reliable estimates are being sought, the larger the sample size that would be needed.

4. Domains/explicit strata (usually chosen based on subjective criteria) increase sample size by a factor of the number of domains. Investigators should therefore give careful thought in treating levels of a given variable as domains because of the multiplicative effect on sample size.

5. Use of clustered sampling rather than a simple random sampling increases the sample size by a factor called the design effect to correct for intra-cluster correlation. The different parameters and assumptions needed for sample size calculation, including typical values, are discussed in Table 2. Sample size calculation is not needed if one performs a complete census of all individuals listed in the sampling frame. The decision to do a census rather than take a sample can be considered in light of the low response rates typical of web surveys, and also given the fact that the incremental cost of recruiting or contacting an additional participant in a web survey is negligible. When a complete census is taken rather than a sample, there are no associated sampling errors, and hence confidence intervals are not warranted when reporting point estimates (confidence intervals are similarly not scientifically justifiable for non-probability samples because there are no sampling errors either).

\section{Sampling or selecting the participants in a} representative manner

This item deals with issues of validity; a probability based sample (e.g. simple random sample) is more valid than a non-probability based sample (e.g. convenience sample). A non-probability sample is so named because selection probabilities are unknown and cannot be numerically calculated for each eligible individual ${ }^{14}$. The underlying basis for probability, i.e. randomization, is absent in nonprobability samples; people opt in or opt out willfully and deliberately (e.g. volunteer samples where individuals self-select into the study, convenience samples where 
Table 2. List of different parameters needed for sample size calculation and their commonly assumed values

\begin{tabular}{|c|c|c|}
\hline Parameter & Name & Illustrative description \\
\hline$D$ & $\begin{array}{l}\text { Number of } \\
\text { domains or } \\
\text { explicit strata }\end{array}$ & $\begin{array}{l}\text { With } n \text { domains, the sample size increases by a factor of } n \text {. Specifying } \\
\text { separate domains or explicit strata ensures adequate size within each } \\
\text { domain. As the different domains or explicit strata can be treated as } \\
\text { completely different populations (an individual can only belong to one } \\
\text { stratum), there is greater sampling flexibility as different sampling } \\
\text { approaches can be used in different strata. Use of domains or explicit strata } \\
\text { increases precision but has no direct impact on validity. }\end{array}$ \\
\hline
\end{tabular}

Deff Design effect $\quad$ Wh $\quad$ De

When clustered sampling is performed rather than simple random sampling, the sample size has to be inflated by a factor called the design effect to correct for intra-cluster correlation. Consider a hypothetical cluster with a high degree of correlation such that every second person is virtually a 'clone' of the first in terms of their responses. It follows that for every two survey responses completed, only one truly unique answer is recorded. Responses from 100 people in this hypothetical cluster corresponds to only 50 truly unique responses and therefore yields estimates with about twice the variance compared to those from a simple random sample of 100 individuals (smaller sample means greater variance). Correcting for this 'clone factor' (formally called the design effect) would require doubling the sample size in the cluster design to 200 to produce the same amount of variance that would have been observed had a simple random sample been taken. Deff = variance estimate (cluster)/variance estimate (simple random sample).

\section{$\varepsilon, \% \quad$ Margin of} error

$\begin{array}{ll}\text { fpc } & \begin{array}{l}\text { Finite } \\ \text { population } \\ \text { correction }\end{array}\end{array}$

Total size of the parent population
The margin of error is half the width of the confidence interval. For example, if a point estimate of $30 \%$ has corresponding $95 \%$ confidence intervals (CI) ranging from $10.4 \%$ to $49.6 \%$, then the width of the $\mathrm{CI}$ is $49.6 \%$ minus $10.4 \%$ $=39.2 \%$. Half of this width (the margin of error) therefore is approximately $20 \%$. Increasingly larger margins of error will yield increasingly imprecise estimates. Conversely, decreasing the margin of error will drastically increase sample sizes. For example, halving the margin of error almost quadruples the required sample size.

The finite population correction factor, $f p c$, is calculated using the formula: $f p c=(N-n) /(N-1)$ where $N$ is the parent population size and $n$ is the computed sample size. As $N$ becomes large relative to the size of $n$, both the numerator $(N-n)$ and the denominator $(N-1)$ tend to $N$, and $f p c$ therefore tends to 1 . The fpc factor only needs to be applied when the sample is a significant proportion of the parent population ( $5 \%$ or higher), otherwise, it is assumed to be 1 . An initial estimate of the sample size is needed to estimate the value of $f p c$.

In general, the size of the sample is independent of the size of the parent population. In other words, the sample size required to compute an overall estimate of an outcome within a county, for example, will invariably be the same as that required for an entire country. The total population size, $N$, influences sample size, $n$, only when the sample constitutes a substantial $(\geq 5 \%)$ proportion of the total population size (i.e. $n / N \geq 0.05$ )

\section{Typical values}

When no explicit strata or domains are being used, $D=1$. Otherwise, $D$ corresponds to the number of mutually exclusive strata.

$1.5-2.0$

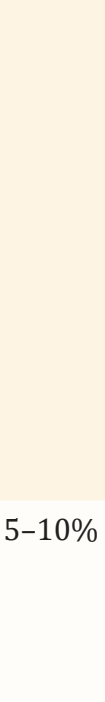

When the sample is much smaller that the parent population $(<5 \%$, as is usually the case), fpc can be taken as 1 .

A rough estimate can be used. For surveys of very large target populations, $\mathrm{N}$ of 1 million would suffice. For surveys of smaller target populations where a finite population correction is more likely to be needed, approximations could be made based on available information. 
Table 2. Continued

\begin{tabular}{|c|c|c|c|}
\hline Parameter & Name & Illustrative description & Typical values \\
\hline$P, \%$ & $\begin{array}{l}\text { Prevalence of } \\
\text { outcome }\end{array}$ & $\begin{array}{l}\text { Since the variance of a binary variable is dependent on the product of } \\
P(1-P) \text {, here } P \text { is used as a fraction, it follows that the variance (and the } \\
\text { corresponding sample size required) is greatest at } 50 \% \text { prevalence. Working } \\
\text { estimates of prevalence of the outcome for the population of interest could } \\
\text { be obtained from the literature as well. }\end{array}$ & $\begin{array}{l}\text { An estimate of the } \\
\text { prevalence could } \\
\text { be obtained from } \\
\text { the literature, or } \\
\text { calculated from } \\
\text { previous survey } \\
\text { waves. If prevalence } \\
\text { is unknown, or } \\
\text { to ensure the } \\
\text { largest sample size } \\
\text { especially with } \\
\text { multiple outcomes, } \\
\mathrm{P}=50 \% \text { could be } \\
\text { used. }\end{array}$ \\
\hline$R, \%$ & $\begin{array}{l}\text { Response } \\
\text { rate }\end{array}$ & $\begin{array}{l}\text { This is the anticipated response rate. By survey mode, in-person surveys } \\
\text { generally have the highest response rates, while remotely completed surveys } \\
\text { such as web and postal mail have the lowest response rates. Adjusting for a } \\
\text { low response rate improves precision, but has no direct impact on validity. }\end{array}$ & $\begin{array}{l}\text { For a web survey } \\
\text { without incentives, } \\
\text { a reasonable } \\
\text { estimate of } \\
\text { anticipated } \\
\text { response rate could } \\
\text { range from } 15 \% \text { to } \\
30 \% \text {. }\end{array}$ \\
\hline$s, \%$ & $\begin{array}{l}\text { Population } \\
\text { size }(\%) \\
\text { of the } \\
\text { smallest base } \\
\text { population }\end{array}$ & $\begin{array}{l}\text { This is the one smallest base population of all the tabulation variables for } \\
\text { which reliable estimates are being sought. To identify the smallest base } \\
\text { population, first identify which tabulation variable has the most levels or } \\
\text { categories, then identify which level has the fewest people. Calculating the } \\
\text { sample size based on the smallest base population guarantees adequate } \\
\text { sample size for every other (bigger) subgroup as well as overall. There is } \\
\text { however a trade-off between wanting reliable estimates for every subgroup } \\
\text { (rather than collapsing certain cells), and the size of the sample required. }\end{array}$ & $\begin{array}{l}\text { If unknown, } 10 \% \\
\text { could be used. }\end{array}$ \\
\hline$Z$ & $\begin{array}{l}\text { Critical } Z \\
\text { value }\end{array}$ & $\begin{array}{l}\text { This statistic measures the distance, measured in standard deviations, a } \\
\text { given observation is from the mean under assumptions of normality. For } \\
\text { example, a } Z \text { statistic of } 0 \text { means that an observation is exactly at the mean, } \\
\text { while a } Z \text { statistic of } 2 \text { means than an observation is } 2 \text { standard deviations } \\
\text { from the mean. The } 68-95-99.7 \text { rule explains that } 68 \%, 95 \% \text { and } 99.7 \% \\
\text { of observations lie approximately within } 1,2 \text {, and } 3 \text { standard deviations, } \\
\text { respectively, from the mean. }\end{array}$ & $\begin{array}{l}Z=1.96 \text {, at the } 95 \% \\
\text { confidence level. }\end{array}$ \\
\hline
\end{tabular}

remote or inaccessible units are deliberately ignored by the investigators, or snowball sampling where existing participants recruit new participants from their own network). Selection probabilities could therefore be zero for certain eligible individuals in a non-probability sample. With probability based samples on the other hand, selection involves randomization; selection probabilities are therefore known, non-zero, and numerically calculable for everyone in the study since both the numerator (respondents) and the denominator (all those eligible) are well defined, positive integers.

While a plethora of non-probability web-based data collection activities exist and do have their role in public health practice (e.g. focus groups, semi-structured interviews, volunteer survey panels ${ }^{19-21}$, probability based sampling is central to inferential surveillance activities. Any one of the numerous approaches to probabilistic sampling (Figures 1 and 2) will theoretically yield valid estimates in the absence of coverage and non-response biases. If a simple random or systematic sampling will be conducted within the context of a web survey, an important first step is to construct a sampling frame - a list of all the sampling units in the study universe, from which the sample will be drawn. Efforts should be made to ensure that the sampling frame is current, correct, and complete.

\section{Sending the survey invitations in a manner that is efficient, safe, and mitigates bias}

Sending large-volume survey invitations en bloc via regular email is likely to get flagged as suspicious or malicious 
activity and blocked by anti-virus software, internet service providers, or corporate mail administrators ${ }^{22}$. In particular, the use of the ' $\mathrm{To}^{\prime}$ ' $\mathrm{Cc}$ ' or 'Bcc' features of emails to invite multiple individuals within the same email should be discouraged as this might lead to unfavorable response rates because of being perceived as spam, or triggering ripple unsubscribe requests. Investigators could use a program with features that allow for batch mailing, customizing delivery to each recipient (e.g. 'Dear John Q. Public' instead of 'Dear participant'), and tracking those who have not responded (to send targeted reminders). Several commercial and open access programs exist for data collection with varying capabilities, including RED Cap (Research Electronic Data Capture; https://www.project-redcap.org/); Epi Info Web Survey (https://www.cdc.gov/epiinfo/cloud.html); Survey Monkey (https://www.surveymonkey.com/); and Google forms (https://www.google.com/forms/about/). A determination of in which environment to host data collection should include a careful consideration of data volume (i.e. total number of respondents), variety (e.g. textonly vs photo/video illustrated questions), vulnerability (e.g. sensitive information and direct identifiers), cost, as well as the software's capabilities (e.g. security controls, ability to implement skip patterns and to filter respondents and non-respondents). Survey administrators should pre-test the survey application on different devices (e.g. computers, smartphones, and tablets) and using different browsers/ browser settings to ensure optimal display across different platforms. Skip patterns, when used, should appear seamless with no apparent discontinuation in the ordering of question numbers from the survey taker's perspective to reduce potential for confusion; alternatively, the entire questionnaire could be unnumbered.

Whenever possible, customized links should be provided for each participant to ensure that each email invitation can only be completed once and by the intended recipient. When only a generic link exists where anyone who has the link can access the survey, recipients should be discouraged from forwarding the survey to any other person. If individuals not in the sampling frame were to be forwarded the survey and complete it, the probability based attributes of the survey begin to get eroded and the survey begins to assume characteristics of a snowball (non-probability) sample.

The consent process could be administered in the introduction page of the web survey; it should be clear to participants that proceeding with the survey indicates that they understand the purpose of the study and agree to participate. The survey team's contact information should also be provided in the introduction page for participants to send questions or concerns about the survey. Completed responses from study participants should be fully delinked from their email or cell phone numbers. Where direct or potential identifiers are being collected, all responses completed online should be done in a secure, electronic, webbased data collection system hosted within an infrastructure where the survey administrators have full control of the data. The survey should have an opening and closing date, after which responses will no longer be recorded. After the survey closing date, data should be transferred from the Server database into a flat database and stored on secured, password protected computers where they will be accessible only to the investigators.

When financial incentives are being used to increase response rates, care should be exercised in the amount offered so that no selection bias is introduced inadvertently (e.g. differential participation rates by individuals of low and high socioeconomic status). Consideration should also be given to the timing of web survey launches and follow-up reminders to ensure optimal response rates. For example, inviting academic faculty or students to complete a web survey during periods of peak school activity (e.g. exam periods) might yield low response rates.

\section{Assessing and enhancing the external validity of collected data}

Two threats to external validity or generalizability in a web survey are poor coverage and low response rates (Figure 4). Poor coverage occurs when the sampling frame does not cover the entire target population. When significant coverage bias exists, a mixed mode survey could be implemented to increase the extent of coverage; for example, a traditional survey mode (e.g. telephone, postal mail, or face-to-face surveys) could be added to the web mode to ensure that everybody in the target population has a known, and nonzero probability of selection. Alternatively, the study population could be redefined (narrowed) such that the areas of non-coverage are excluded.

Testing for non-response bias is especially important in web surveys because of the typically high rates of nonresponse. A validation survey which involves conducting a follow-up survey on a sample of non-respondents, would typically be needed to empirically test differences between respondents and non-respondents. Intensive efforts are often needed to elicit a response from original nonrespondents within validation surveys, including use of incentives, using a shortened version of the questionnaire to encourage response, and using additional ways to contact participants, beyond merely emailing, e.g. direct mail, phone calls or in-person visit. Besides helping to detect non-response, a validation survey can help mitigate nonresponse bias, while also increasing the effective response rate. Several scientific journals will only publish findings from surveys with 'high' response rates, as such there is a strong motivation to increase the effective response rate of low-response web surveys.

Where necessary, different types of weights, including sampling weights (inverse of selection probabilities), non-response weights (inverse of the response rate), and calibration weights (standardizing the demographic distributions of the sample to that of the parent population) 
Figure 4. Relationships between the target, sampled, and analyzed populations in a survey

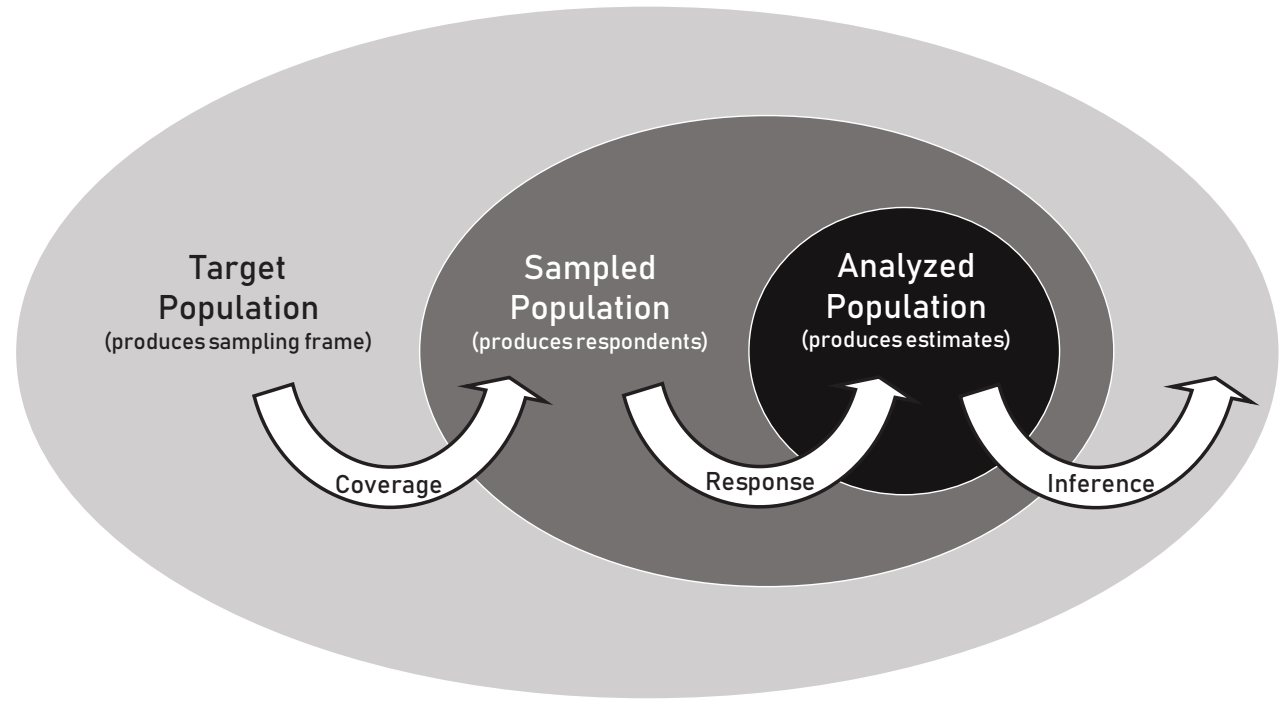

Figure 5. Anatomy of survey weights used to adjust for differential selection probabilities and response rates, and to calibrate the sample to the standard population

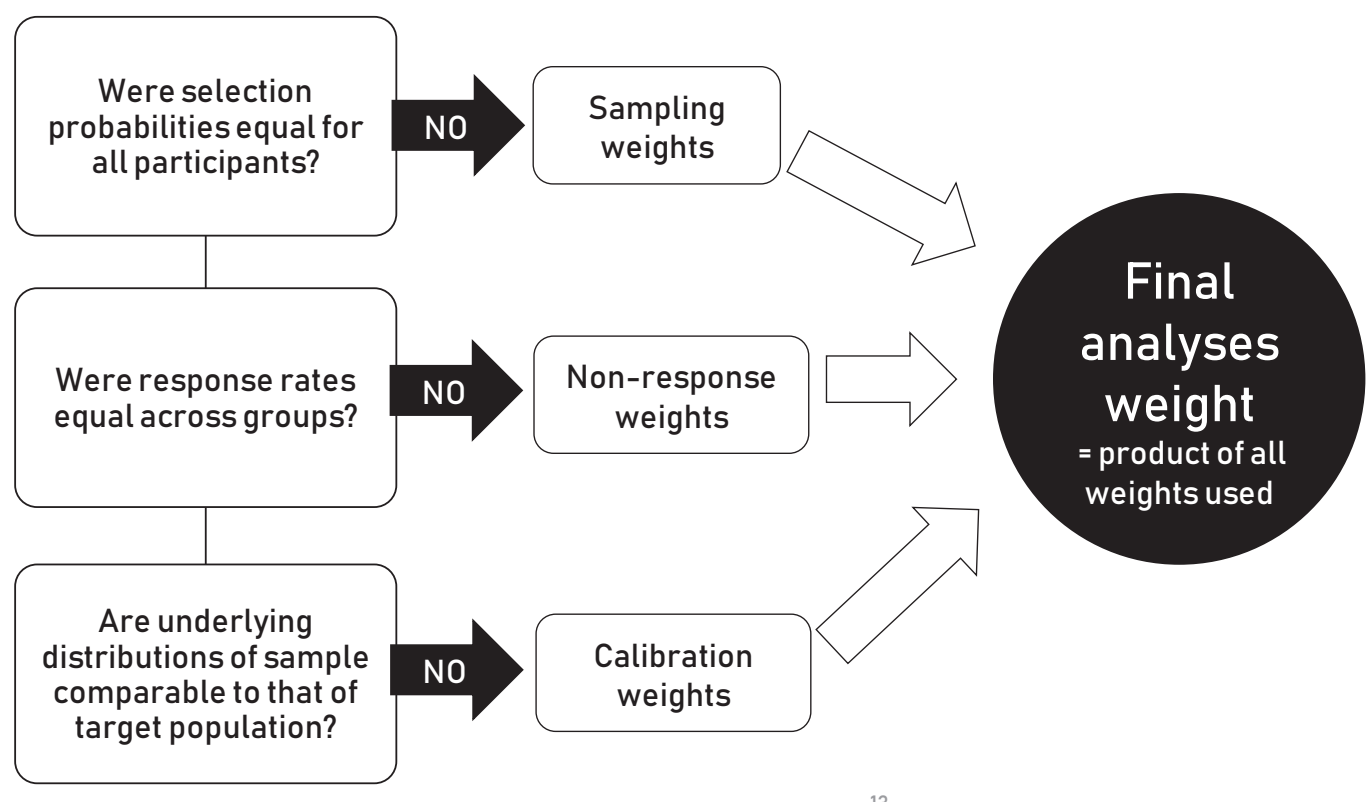

may be used to enhance the external validity of a survey (Figure 5). All, none or a variable number of these weights may be applied depending on sampling approach used, patterns of non-response observed, and the availability of data on sampling frame characteristics. For example, sampling weights only apply when selection probabilities are differential (e.g. oversampling of certain groups); they do not apply when selection probabilities are equal for everyone (e.g. simple random, or systematic sampling). Similarly, non-response weights only apply when nonresponse rates are differential across groups (i.e. not when all groups have comparably high or comparably low response rates). Calibration weights can only be created when data are available on the demographic distribution of the parent population. The final analysis weight is computed as the product of the individual weights used.

\section{DISCUSSION}

Although web surveys in general are becoming increasingly easier to perform, even without formal training in survey methodology, good web surveys in contrast are becoming increasingly harder to conduct (as measured by indexes such as response rate and coverage ${ }^{7}$. Nonetheless, web surveys can play an important role in public health by providing a mechanism to surveil trending issues of public health importance in near real-time, for program and policy 
action. Their flexibility can easily allow incorporation of certain features that may be cumbersome in traditional survey modes, including such features as videos, pictures, and images ${ }^{23}$.

Determining the purpose for which data are being collected online is an important first step in survey design as this undergirds the assumptions made and defines the nature of the information collection activity ${ }^{23}$. For example, if the purpose is to perform an online experiment where individuals are randomized to two or more interventions (e.g. different health communication messages) and shortterm outcomes such as cognitions and emotions measured, then a volunteer sample could well be used (randomized trials have strong internal validity, but weak external validity because of their non-probabilistic sampling). In contrast, if the purpose is to estimate a population parameter from the sample, then, special attention should be given to issues of generalizability (cross-sectional surveys have strong external validity, but weak internal validity because of temporality bias and measurement errors).

\section{CONCLUSIONS}

Careful consideration should be given to sampling and nonsampling sources of error when designing web surveys to ensure validity and reliability. While this article does not discuss several technical issues that are beyond its scope, it nonetheless provides survey design principles, resources, and tools that could assist public health practitioners and researchers when implementing web-based surveys.

\section{REFERENCES}

1. Wang TW, Lemos PR, McNabb S, King BA. Attitudes Toward Smoke-Free Public Housing Among U.S. Adults, 2016. Am J Prev Med. 2018;54(1):113-118. doi:10.1016/j.amepre.2017.08.026

2. Liu LL, Li TM, Teo AR, Kato TA, Wong PW. Harnessing Social Media to Explore Youth Social Withdrawal in Three Major Cities in China: Cross-Sectional Web Survey. JMIR Ment Health. 2018;5(2):e34. doi:10.2196/mental.8509

3. Dyar OJ, Nathwani D, Monnet DL, Gyssens IC, Stålsby Lundborg C, Pulcini C; ESGAP Student-PREPARE Working Group . Do medical students feel prepared to prescribe antibiotics responsibly? Results from a cross-sectional survey in 29 European countries. J Antimicrob Chemother. 2018;73(8):2236-2242. doi:10.1093/jac/dky150

4. Manana PN, Kuonza L, Musekiwa A, Koornhof H, Nanoo A, Ismail N. Feasibility of using postal and web-based surveys to estimate the prevalence of tuberculosis among health care workers in South Africa. PLoS One. 2018;13(5):e0197022. doi:10.1371/journal.pone.0197022

5. Borodovsky JT, Marsch LA, Budney AJ. Studying Cannabis Use Behaviors With Facebook and Web Surveys: Methods and Insights. JMIR Public Health Surveill. 2018;4(2):e48. doi:10.2196/publichealth.9408

6. da Costa FF, Schmoelz CP, Davies VF, Di Pietro PF, Kupek
E, de Assis MA. Assessment of diet and physical activity of brazilian schoolchildren: usability testing of a webbased questionnaire. JMIR Res Protoc. 2013;2(2):e31. doi:10.2196/resprot.2646

7. Couper MP. Web Surveys - a review of Issues and approaches. Public Opinion Quarterly. 2020;64:464-494. doi:10.1086/318641

8. Craig S. 90 Interesting facts about email: How many emails are sent per day? DMR Business Statistics. Updated July 11, 2020. Accessed May 15, 2018. https://expandedramblings. com/index.php/email-statistics/

9. Jones MK, Calzavara L, Allman D, Worthington CA, Tyndall M, Iveniuk J. A Comparison of Web and Telephone Responses From a National HIV and AIDS Survey. JMIR Public Health Surveill. 2016;2(2):e37. doi:10.2196/publichealth.5184

10. Yang MC, Tan EC. Web-based and telephone surveys to assess public perception toward the national health insurance in taiwan: a comparison of cost and results. Interact J Med Res. 2015;4(2):e9. doi:10.2196/ijmr.4090

11. Brøgger J, Nystad W, Cappelen I, Bakke P. No increase in response rate by adding a web response option to a postal population survey: a randomized trial. J Med Internet Res. 2007;9(5):e40. doi:10.2196/jmir.9.5.e40

12. McMahon SR, Iwamoto M, Massoudi MS, et al. Comparison of e-mail, fax, and postal surveys of pediatricians. Pediatrics. 2003;111(4 Pt 1):e299-303. doi:10.1542/peds.111.4.e299

13. Milton AC, Ellis LA, Davenport TA, Burns JM, Hickie IB. Comparison of Self-Reported Telephone Interviewing and Web-Based Survey Responses: Findings From the Second Australian Young and Well National Survey. JMIR Ment Health. 2017;4(3):e37. doi:10.2196/mental.8222

14. United Nations. Expert Group Meeting to Review the Draft Handbook on Designing of Household Sample Surveys. United Nations Statistics Division; 2003. ESA/STAT/ AC.93/L.1. Accessed May 15, 2018. https://unstats.un.org/ unsd/demographic/meetings/egm/sampling_1203/docs/ 11.pdf

15. Association of Health Care Journalists. Understanding HIPAA: A brief overview. Accessed September 22, 2018. https:// healthjournalism.org/resources-tips-details.php?id=12\#. YTdVFY4zZPY

16. U.S. Department of Health \& Human Services. Summary of the HIPAA Privacy Rule. Accessed March 19, 2019. https://www. hhs.gov/hipaa/for-professionals/privacy/laws-regulations/ index.html

17. U.S. Department of Health \& Human Services. Regulation 45 CFR 46. Accessed March 19, 2019. https://www.hhs.gov/ ohrp/regulations-and-policy/regulations/45-cfr-46/index. html

18. Centers for Disease Control and Prevention. Distinguishing Public Health Research and Public Health Non Research. July 29, 2010. Accessed March 19, 2019. https://www.cdc.gov/ os/integrity/docs/cdc-policy-distinguishing-public-healthresearch-nonresearch.pdf

19. Sharma A, O'Connor R, Celestino P, Killion S, Griswold- 
Krupski L, Bansal-Travers M. Focus Groups and In-depth Interviews to Guide the Development of Lung Cancer Screening Informational Materials. J Cancer Educ. 2018;34(4):712-718. doi:10.1007/s13187-018-1362-4

20. Camenga DR, Fiellin LE, Pendergrass T, Miller E, Pentz MA, Hieftje K. Adolescents' perceptions of flavored tobacco products, including E-cigarettes: A qualitative study to inform FDA tobacco education efforts through videogames. Addict Behav. 2018;82:189-194. doi:10.1016/j.addbeh.2018.03.021

21. Kaufman P, Kang J, Kennedy RD, Beck P, Ferrence R. Impact of smoke-free housing policy lease exemptions on compliance, enforcement and smoking behavior: A qualitative study. Prev Med Rep. 2018;10:29-36. doi:10.1016/j.pmedr.2018.01.011 22. Microsoft. Too many recipients. MSOutlook.info. Accessed Marh 19, 2019. https://www.msoutlook.info/question/198 23. Wyatt JC. When to Use Web-based Surveys. J Am Med Inform Assoc. 2000;7(4):426-430. doi:10.1136/jamia.2000.0070426

\section{CONFLICTS OF INTEREST}

The author has completed and submitted the ICMJE Form for Disclosure of Potential Conflicts of Interest and none was reported.

FUNDING

There was no source of funding for this research.

ETHICAL APPROVAL AND INFORMED CONSENT

Ethical approval and informed consent were not required for this study.

\section{DATA AVAILABILITY}

Data sharing is not applicable to this article as no new data were created.

PROVENANCE AND PEER REVIEW

Not commissioned; externally peer reviewed. 